Helicobacter pylori

\section{Aspirin injury and $\mathrm{H}$ pylori}

\section{A Schmassmann}

\section{The role of Helicobacter pylori in aspirin induced gastric injury}

Helicobacter pylori and non-steroidal antiinflammatory drugs (NSAIDs) cause the vast majority of peptic ulcers and their complications. However, their interaction remains extremely controversial. The study of Yoshida et al in this issue of $G u t^{1}$ aimed to address this issue by investigating the influence of experimental $\mathrm{H}$ pylori infection on gastric mucosal injury induced by aspirin in male Mongolian gerbils [see page 594].

The study found that aspirin caused more extensive haemorrhagic erosions associated with greater myeloperoxidase activity (an index of neutrophil accumulation), thiobarbituric acid reactive substance concentrations (an index of lipid peroxidation), and $\mathrm{KC} / \mathrm{GRO}$ concentrations (a chemoattractive cytokine in rodents) in infected than in uninfected gerbils.

Furthermore, the authors repeated the experiments in gerbils pretreated with antineutrophil serum which reduced circulating neutrophils by $77 \%$. Erosion index, myeloperoxidase activity, and thiobarbituric acid reactive substance concentration (but not KC/GRO concentration) were significantly less in such neutrophil depleted gerbils exposed to $H$ pylori plus aspirin than in similarly exposed animals treated with control serum. Inhibitory effects on mucosal damage by antineutrophil serum administration was much greater in $\mathrm{H}$ pylori infected gerbils (65\%) than in uninfected gerbils $(31 \%)$. Yoshida et al concluded that $H$ pylori infection potentiates aspirin induced gastric mucosal injury by mechanisms that include accumulation of activated neutrophils.

This is one of the first experimental studies showing convincingly that aspirin induced gastric injury is aggravated by $H$ pylori infection. Furthermore, Yoshida et al give strong support that $H$ pylori potentiates aspirin injury via a neutrophil dependent process. However, the study should be completed by proving that $H$ pylori eradication will reverse the severity of aspirin induced gastric injury to levels found in uninfected gerbils. The data of Yoshida and colleagues ${ }^{1}$ are well in line with recent experimental studies which have indicated that neutrophil adherence to the endothelium via various adhesion molecules is involved in the development of gastric mucosal injury induced by $H$ pylori infection or NSAID use. $^{23}$ Activated neutrophils have been suggested to injure endothelial and epithelial cells by producing active oxygen species and proteases. ${ }^{4}$ These data suggest that $H$ pylori and NSAIDs can cause an acute inflammatory response in the gastric mucosa leading to neutrophil mediated injury. ${ }^{2-4}$

Yoshida and colleagues ${ }^{1}$ performed their studies in Mongolian gerbils infected with $H$ pylori. These infected animals develop pathological changes in the stomach that mimic those seen in humans infected by $H$ pylori. About three weeks after $H$ pylori inoculation, gerbils exhibit typical gastritis with neutrophil and mononuclear cell infiltration in the lamina propria. The gerbils subsequently show chronic gastritis (including formation of lymphoid follicles) approximately six weeks after $H$ pylori infection and develop gastric ulcers at more than six months after infection. This Mongolian gerbil model may allow, in additional studies, assessment of the role of $\mathrm{H}$ pylori on NSAID induced ulcer development. It would be particularly interesting to assess the role of $\mathrm{H}$ pylori eradication in gerbils six months after infection before experimental long term treatment with NSAIDs.

What to do about $H$ pylori in NSAID users who are infected with the organism poses a difficult therapeutic dilemma, which continues to be controversial. Whether $H$ pylori infection affects the outcome of NSAID therapy in humans is only partly clear at the present time. ${ }^{5-8} H$ pylori gastritis seems to increase the likelihood of developing dyspeptic symptoms in patients on NSAID therapy. In addition, there is evidence that the histological severity of $H$ pylori gastritis may be adversely affected by NSAID therapy, with a consequent increase in the risk of developing a peptic ulcer, possibly with complications. Furthermore, ulcers are more likely to develop during the course of NSAID therapy in those infected with $H$ pylori; eradication of the infection reduces ulcer recurrence in the face of continued NSAID therapy, and it seems likely that this must reduce the risk of gastrointestinal bleeding in those using NSAIDs. The effect of $H$ pylori infection on the risk of perforation during NSAID therapy is unclear at the present time.

The mechanisms of NSAID injury to the gastrointestinal tract are complex and not mediated solely by activated neutrophils; several other mechanisms may be involved such as inhibition of prostaglandin and thromboxane synthesis, damage to blood vessels, inhibition of repair mechanisms, increase in gastrointestinal permeability, drug entrapment in gastric cells, uncoupling of oxidative phosphorylation in mitochondria, loss of cytoskeletal control over tight junction, decrease in gel hydrophobicity, inhibition of phospholipase, and interaction with inducible nitric oxide synthase and nitric oxide. ${ }^{9-11}$ In contrast, the effects of $H$ pylori on the gastrointestinal tract differ substantially from these NSAID related mechanisms. In particular, NSAIDs and $H$ pylori have opposite effects on prostaglandin synthesis; NSAIDs decrease whereas $H$ pylori increases prostaglandin synthesis in the gastric mucosa. The modest stimulatory effects of $H$ pylori on prostaglandin (and nitric oxide) synthesis are unlikely to confer significant protection in the presence of NSAIDs as $H$ pylori also produces a broad spectrum of pathophysiological changes-for example, reduction of viscosity of mucus gel facilitating back diffusion of hydrogen ions and reduction of mucosal blood flow which have the potential to diminish the resistance of the gastric mucosa to NSAID exposure. Additionally, gastric acid secretion, which is increased in the majority of $H$ pylori infected subjects, favours gastric mucosal damage as the severity of NSAID damage is dependent on gastric $\mathrm{pH}$.

In the future, it is possible that the impact of the questions mentioned above will decline because of the decreasing prevalence of $H$ pylori infection in the developed world and the increasing use of cyclooxygenase 2 inhibitors and new specific inhibitors of thrombocyte aggregation (such as clopidogrel) which neither appear to cause ulcers nor to interact significantly with $H$ pylori.

Gut 2002;50:589-590

\section{Author's affiliation}

A Schmassmann, Department of Medicine, KSSW Lucerne, 6210 Sursee, Switzerland

\section{Correspondence to:}

Adrian.Schmassmann@kssw.ch

\section{REFERENCES}

1 Yoshida N, Sugimoto N, Hirayama F, et al. Helicobacter pylori infection potentiates aspirin induced gastric mucosal injury in Mongolian gerbils. Gut 2002;50:594-9

2 Wallace JL, Arfors KE, McKnight GW. A monoclonal antibody against the CD18 leukocyte adhesion molecule prevents indomethacin-induced gastric damage in the rabbit. Gastroenterology 1991;100:878-83.

3 Taha AS, Dahill S, Morran C, et al. Neutrophils, Helicobacter pylori, and non-steroidal anti-inflammatory drug ulcers. Gastroenterology 1999;1 16:254-58. 
4 Suzuki M, Miura S, Suematsu $M$, et al. Helicobacter pylori-associated ammonia production enhances neutrophil-dependent gastric mucosal injury. Am J Physiol 1992;263:G719-25.

5 Chan FK, Sung JJ, Chung SC, et al. Randomised trial of eradication of Helicobacter pylori before non-steroida anti-inflammatory drug therapy to prevent peptic ulcers. Lancet 1997:350:975-9.

6 Hawkey CJ, Tulassy Z, Szczepanski L, et al. A randomised controlled trial of Helicobacter pylori eradication in patients taking non-steroidal anti-inflammatroy drugs: the
HEIP NSAIDs study Lancet 1998;352:1016-21.

7 Aalykke C, Laurtisen JM, Hallas J, et al. Helicobacter pylori and risk of ulcer bleeding among users of nonsteroidal anti-inflammatory drugs: a case-control study. Gastroenterology drugs: a case-control

8 Chan FK, Chung SC, Suen BY, et al. Preventing recurrent upper gastrointestinal bleeding in patients with Helicobacter pylori infection who are taking low-dose aspirin or naproxen. N Engl J Med 2001;345:67-8.

9 Schmassmann A, Tarnawski A, Peskar BM, et al. Influence of acid and angiogenesis on

\section{The SPINK in chronic pancreatitis: similar finds, different minds}

\section{H Witt}

\section{SPINK mutations are strongly associated with chronic pancreatitis but may not cause the disease}

\author{
Student \\ Mephistopheles \\ "Yet in each word some concept there must be." \\ "Quite true! But don't torment yourself too anxiously; \\ For at the point where concepts fail, \\ At the right time a word is thrust in there. \\ With words we fitly can our foes assail, \\ With words a system we prepare, \\ Words we quite fitly can believe, \\ Nor from a word a mere iota thieve."
}

Johann Wolfgang Goethe; Faust I

Approximately five decades ago it was recognised for the first time that chronic pancreatitis $(\mathrm{CP})$ may cluster in selected families suggesting an inherited disorder in these patients. ${ }^{1}$ However, the underlying genetic defect remained obscure for a long time. The discovery of cationic trypsinogen (PRSS1) mutations in $\mathrm{CP}$ families provided a first clue to the underlying disease mechanisms. ${ }^{2}$ Subsequent studies revealed that PRSSI mutations are also present in patients with idiopathic CP. Recently, mutations in the serine protease inhibitor, Kazal type 1 (SPINK1) gene, a pancreatic trypsin inhibitor, were identified as associated with idiopathic or hereditary CP: in 22 of 96 paediatric patients, a SPINK1 mutation was detected. In 18 patients, a substitution of asparagine by serine at codon 34 in exon 3 was found (N34S). Six patients were homozygous for this mutation. ${ }^{3}$ These findings suggest that pancreatitis may be the result of an imbalance of proteases and their inhibitors within the pancreatic parenchyma.
In this issue of Gut, Threadgold and coworkers $^{4}$ and Drenth and colleagues describe mutational studies of SPINK1 in two large series with different types of chronic pancreatitis [see pages 675 and 682]. Threadgold et al found the SPINK1 N34S mutation in 4/108 (3.7\%) patients with hereditary CP, in 6/7 (86\%) cases of so-called "familial idiopathic $\mathrm{CP}^{\prime \prime}$, in $11 / 87(13 \%)$ patients with socalled "true idiopathic CP", and in 4/67 (6\%) patients with alcohol related $\mathrm{CP}^{4}$ Drenth et al detected an N34S mutation in $2 / 10(20 \%)$ patients with hereditary $\mathrm{CP}$, in $5 / 24(21 \%)$ patients with idiopathic CP, in 4/72 (5.6\%) cases with alcohol induced CP, and in 2/9 (22\%) patients with a miscellaneous origin of CP.

Both studies showed a strong association between N34S and various types of $\mathrm{CP}$ but the mutation frequencies reported in different $\mathrm{CP}$ groups and the interpretation of their data differ markedly. In hereditary CP patients, Drenth et al found the N34S mutation in 20\% whereas Threadgold et al detected this kinetics of gastric ulcer healing in rats: interaction with indomethacin. Am J Physiol 1995;268:G276-85

10 Schmassmann A, Peskar BM, Stettler C, et al. Effects of inhibition of prostaglandin endoperoxidase synthase-2 in chronic gastro-intestinal ulcer models in rats. $\mathrm{Br} \mathrm{J}$ Pharmacol 1998;123:795-804.

11 Halter F, Peskar BM, Schmassmann A, et al. Cyclooxygenase 2-implications on maintenance of gastric mucosal integrity and ulcer healing: controversial issues and perspectives. Gut 2001;49:443-53. mutation in only $3.9 \%$. Previous studies showed an N34S frequency in hereditary CP patients of $9.1 \%{ }^{6}$ and $7.0 \%{ }^{7}$ These considerable differences may be partially explained by the fact that in some studies the N34S frequency was calculated by counting several members of one family whereas in other studies the frequency was determined by counting unrelated patients only. Furthermore, each group used different definitions of hereditary and idiopathic CP. Drenth et al diagnosed hereditary CP on the basis of two or more affected family members whereas Threadgold et al made the diagnosis of hereditary $\mathrm{CP}$ on the basis of two affected first degree or three or more affected second degree relatives in two or more generations. Thus in the latter study several patients with a family history where classified as having "familial" idiopathic CP. The concept of "familial" and "true" idiopathic CP appears strange as the term idiopathic usually implies the absence of any predisposing factor, including heredity. The chosen contradictio in adjecto "familial idiopathic" throws light on different understandings of hereditary $\mathrm{CP}$ and reflects the lack of a uniform terminology. As the first description of inherited pancreatitis suggested an autosomal dominant inheritance, ${ }^{1}$ hereditary CP was defined as a dominant inherited disease. Subsequently, the finding of familial clustering in one generation only, which indicates other inheritance patterns such as recessive or complex trait, was blinded out in the disease concept of hereditary CP.

The observed N34S frequencies in alcohol related CP were similar in both studies published in this issue of Gut (6.0\% and $5.6 \%$, respectively) and are in line with the previously reported frequency of $5.8 \%{ }^{8}$ In contrast with Drenth et al however, Threadgold et al failed to find a significant association between N34S and alcoholic CP due to the high N34S frequency in their control subjects from the Liverpool area (4/100).

Interestingly, Drenth et al also found SPINK1 mutations in individuals in which the cause of $\mathrm{CP}$ was attributed to metabolic disorders or anatomical 
anomalies. Although the number of investigated cases was small $(n=9)$ and thus their results might be only preliminary, these data open exciting new research directions. Future investigations have to show whether genetic variations influence disease susceptibility or severity in patients with anatomical variations, abdominal trauma, or other factors. Probably future studies may show a complex relationship between multiple genetic and environmental factors with different weights of these factors depending on the type of CP.

Both articles in this issue of Gut reflect the ongoing controversy on the meaning and inheritance pattern of SPINKI mutations in CP. It was argued that patients with mutant SPINKl could not suffer from autosomal recessive disease because individuals who are heterozygous for N34S ought to be asymptomatic. However, in two previous studies, approximately $10 \%$ of patients with idiopathic CP were homozygous for N34S. ${ }^{36}$ Assuming an N34S carrier frequency of about $1-2 \%$ in the general population, the observed frequency of N34S homozygotes was 1000-4000 times higher than expected. Although a recessive inheritance pattern does not explain the high frequency of observed N34S heterozygotes, refusing a recessive trait does not explain the strong accumulation of N34S homozygotes in CP.

There is now growing evidence that many inherited disorders of the gastrointestinal tract such as haemochromatosis, $\alpha_{1}$ antitrypsin deficiency, or CP follow a more complex trait and not only simple mendelian inheritance patterns: for example, hereditary haemochromatosis is caused in most cases by a cysteine to tyrosine substitution at position 282 (C282Y) in the HFE gene. Several C282Y homozygotes however do not suffer from clinical apparent liver disease. Moreover, a significant percentage with symptomatic haemochromatosis are only heterozygous for $\mathrm{C} 282$ Y or bear no $H F E$ mutation, indicating that other genes are involved also. This is supported by the finding of transferrin receptor 2 mutations in haemochromatosis families. Furthermore, recent research showed that haemochromatosis may also follow an autosomal dominant trait caused by ferroportin mutations. In summary, haemochromatosis may be induced by different recessive, dominant, and complex mechanisms in different genes. The same is clearly true for CP. One may discuss if the terms dominant or recessive are appropriate for these diseases, however they may be helpful to navigate through the genetic sea, keeping in mind that all models are only coordinate systems but never image exactly the reality.

Discussion of the role of SPINKI in CP (disease inducer, per se, modifier) reflects the lack of sufficient knowledge of these as yet unidentified factors and does not contribute usefully to the understanding of inherited CP. Usually, the first step is to identify the disease causing gene or genes and later to isolate modifying genes. Different mutations in different genes may have different inheritance patterns. For example, PRSSI mutations are thought to be dominant. However, the PRSS1 Al6V mutation is mainly found in idiopathic CP.

As the majority of patients with idiopathic or hereditary CP do not show a SPINK1 or PRSS1 mutation, ${ }^{3}$ several other genes may be involved in the disease pathogenesis. Future research will identify more pancreatitis related genes and will also more precisely determine the meaning of mutations in the cystic fibrosis transmembrane conductance regulator (CFTR) gene in $\mathrm{CP}^{9}{ }^{10}$ Several studies found a CFTR mutation in up to $30 \%$ of patients with idiopathic CP. In contrast with Threadgold et al who proposed that CFTR is not a good candidate gene for the second gene in patients with SPINKl mutations, we assume consistent with the opinion of Drenth et al, that CFTR mutations have a major impact on the disease pathogenesis. Possibly, in a more sophisticated way, three or more different genetic defects lead to CP. Future genetic studies on CFTR and other candidate genes will give important insights into the mechanisms of inherited pancreatitis and will probably lead to a complex genetic model.

Gut 2002;50:590-591

\section{Author's affiliation}

H Witt, Kinderklinik, Charité-Campus

Virchow-Klinikum, Humboldt-Universität, Augustenburger Platz 1, D-13353 Berlin, Germany; heiko.witt@charite.de

\section{REFERENCES}

1 Comfort MW, Steinberg AG. Pedigree of a family with hereditary chronic relapsing pancreatitis. Gastroenterology 1952;21:54-63

2 Whitcomb DC, Gorry MC, Preston RA, et al. Hereditary pancreatitis is caused by a mutation in the cationic trypsinogen gene. Nat Genet 1996;14:141-5.

3 Witt $\mathbf{H}$, Luck W, Hennies $\mathrm{HC}$, et al. Mutations in the gene encoding the serine protease inhibitor, Kazal type 1 are associated with chronic pancreatitis. Nat Genet 2000;25:213-6.

4 Threadgold J, Greenhalf W, Ellis I, et al. The N34S mutation of SPINK 1 (PSTI) is associated with a familial pattern of idiopathic chronic pancreatitis but does not cause the disease. Gut 2002:50:675-82.

5 Drenth JPH, te Morsche R, Jansen JBM. Mutations in the serine protease inhibitor Kazal type 1 are strongly associated with chronic pancreatitis. Gut 2002;50:687-93.

6 Pfützer RH, Barmada NM, Brunskill APJ, et al. SPINK 1/PSTI polymorphisms act as disease modifiers in familial and idiopathic chronic pancreatitis. Gastroenterology 2000;1 19:615-23

7 Witt H. Gene mutations in children with chronic pancreatitis. Pancreatology $2001 ; 1: 432-8$

8 Witt H, Luck W, Becker M, et al. Mutation in the SPINK 1 trypsin inhibitor gene, alcohol use and chronic pancreatitis. JAMA 2001:285:2716-7.

9 Sharer N, Schwarz M, Malone G, et al. Mutations of the cystic fibrosis gene in patients with chronic pancreatitis. N Engl J Med 1998:339:645-52

10 Cohn JA, Friedman KJ, Noone PG, et al. Relation between mutations of the cystic fibrosis gene and idiopathic pancreatitis. $N$ Engl J Med 1998;339:653-8. 